\title{
Isolated corpus spongiosum injury after sexual intercourse
}

\author{
Ioannis Anastasiou, Aikaterini Anastasiou, Ioannis Katafigiotis, Dimitrios Tsavdaris, \\ Constantinos Constantinides \\ $1^{\text {st }}$ Urology Department, Laiko Hospital, University of Athens, Athens, Greece.
}

\begin{abstract}
Summary
Penile fractures are generally rare and underreported. The mechanism of injury is due to a rupture of the corpora cavernosa following blunt or sexual trauma to the penis when fully erect. Penile fractures usually present with a 'popping' sound with concomitant sudden swelling and ecchymosis of the penis followed by rapid detumescence. Urethral involvement occurs only in a small part of the cases. Isolated spongiosal injury after sexual intercourse is also extremely rare. The cardinal sign of urethral injury is blood at the meatus. A small laceration can be repaired by simple closure with absorbable sutures, while a complete rupture requires a more complex anastomotic repair. We report a case of a typically presenting penile fracture that was eventually proven to be an isolated corpus spongiosum injury, with no corpora cavernosa involvement.
\end{abstract}

KEY WORDS: Penile fracture, Urethral injury, Urological trauma, Urethroplasty.

Submitted 6 March 2018; Accepted 7 May 2018

\section{INTRODUCTION}

Penile fractures are generally rare and underreported. The mechanism of injury is due to a rupture of the corpora cavernosa following blunt or sexual trauma to the penis when fully erect (1). Penile fractures usually present with a 'popping' sound with concomitant sudden swelling and ecchymosis of the penis followed by rapid detumescence. Urethral involvement happens only at a small percentage of the cases (1). Symptoms from the urinary tract include blood at the meatus and subsequently a positive urinalysis for blood and/or acute urinary retention (2). We report a case of a typically presenting penile fracture that was eventually proven to be an isolated corpus spongiosum injury, with no corpora cavernosa involvement.

\section{Case report}

A 47-year-old man visited the emergency department of our hospital complaining of severe pain and a swollen penis shortly after sexual intercourse the previous night. His medical and surgical history was unremarkable. He mentioned a sudden pain on his penis followed by prompt detumescence after failing to enter his partner's vagina and hitting with his partially loose penis on her buttocks. He passed blood-stained urine mainly at the beginning of the stream, but was otherwise able to void.
Subsequently, he presented at the emergency department 6 hours after the accident. During physical examination he presented with a flaccid edematous penis with ecchymosis and blood at the meatus. There was marked tenderness on palpation of the ventral surface of the penis but no palpable abnormalities were noted (Figure 1). The patient was able to void. A complete blood count, serum electrolytes revealed no abnormalities and urine analysis revealed haematuria. Our patient was scheduled for surgery in order to repair what obviously appeared as a penile fracture using a degloving incision. During surgical exploration no recognizable trauma of the corpora cavernosa was demonstrated so we decided to create an artificial erection, which confirmed our findings. A hematoma was discovered at the abdominal surface of the shaft that was lying over a tear in the corpus spongiosum at the level of the penile urethra approximately $4 \mathrm{~cm}$ from the glans. The urethra appeared to be torn with the Foley catheter easily shown underneath. (Figure 1). The defect was closed primarily with 3-0 absorbable sutures, with the Foley catheter in place. The urethral catheter remained for 2 weeks. The patient returned six weeks later fully recovered and able to achieve micturition with no problems whatsoever.

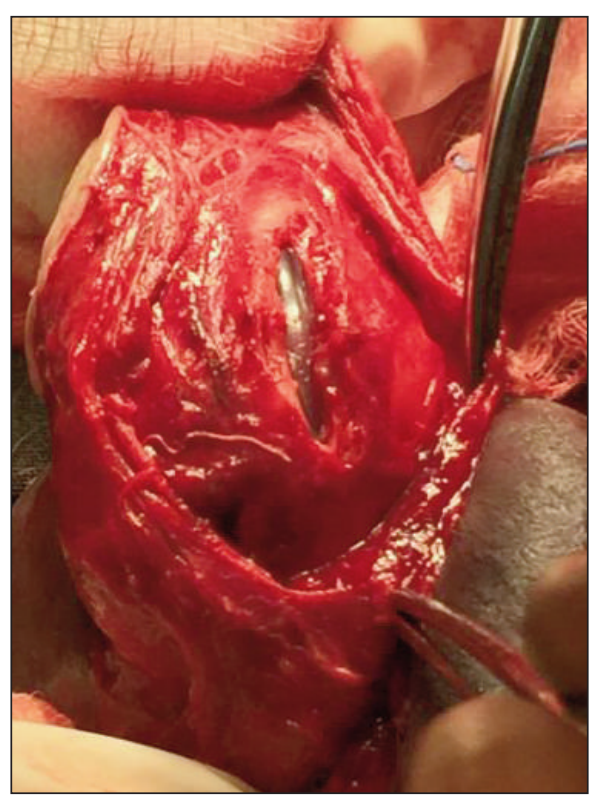

Figure 1. Isolated corpus spongiosum injury. 


\section{Discussion}

Our patient showed typical clinical characteristics of blunt cavernosal trauma with concomitant urethral involvement. However, it was eventually discovered surgically that he suffered an isolated injury to the corpus spongiosum. In our patient, the diagnosis of penile fracture was made due to the typical clinical features he presented with. No further imaging was performed because surgical exploration was already indicated and surgical repair was probably required. The typical mechanism of injury is during sexual intercourse when the penis fails to enter the vagina and strikes against the pubic symphisis or perineum of the partner involved. At the time of injury a rupture of the cavernosal tunica albuginea occurs with subsequent subcutaneous hematoma of the shaft and involvement of the corpus spongiosum or urethra in approximately $10-22 \%$ of cases (1). Isolated spongiosal injury after sexual intercourse is extremely rare.

Blood at the meatus is the cardinal sign of urethral injury and signifies the necessity for further evaluation.

In the case of a complete urethral rupture acute urinary retention is the rule with evidence of a palpable distended bladder (2). Other signs and symptoms of urethral trauma include hematuria, pain while urinating, and scrotal, penile or perineal edema and ecchymosis due to urinary extravasation and bleeding which can present spontaneously or with a several hour delay (2).

Penile fractures require immediate exploration because delayed treatment results in higher rates of erectile dysfunction (3).

The surgical technique consists of closing the tear in the cavernosal tunica albuginea as well as the concomitant tear in the urethra (3). A small laceration can be repaired by simple closure with absorbable sutures, while a complete rupture requires a more complex anastomotic repair (3).

If a concomitant urethral injury is suspected, the gold standard approach for diagnostic evaluation is a retrograde urethrogram (RUG). An alternative and more frequently used examination is the flexible cystoscopy under anesthesia during exploration/ repair.

\section{Conclusions}

The common presentation of sexual trauma is penile fracture. The majority of the cases have a typical etiology of a penis collision against the perineum or the symphysis pubis. The urologist that encounters a patient with a sexual trauma usually manages the sexual trauma as a common penile fracture and an isolated spongiosal injury could be easily missed. It is important for the urologist that deals with this emergency situation to be aware of the possibility of urethral injury even though the penis is integral, especially when there is blood at the meatus (cardinal sign) or inability to void.

\section{REFERENCES}

1. Nicolaisen GS, et al. Rupture of the corpus cavernosum: surgical management. J Urol. 1983; 130:917.

2. Mundy AR, et al. Urethral trauma. Part I: introduction, history, anatomy, pathology, assessment and emergency management. BJU Int. 2011; 108:310.

3. Derouiche A, et al. Management of penile fractures complicated by urethral rupture. Int J Impot Res. 2008; 20:111.

\section{Correspondence}

Ioannis Anastasiou, MD

ekati2@otenet.gr

Aikaterini Anastasiou, MD (Corresponding Author)

aikatianast@gmail.com

Ioannis Katafigiotis, MD

katafigiotis.giannis@gmail.com

Dimitrios Tsavdaris, MD

dimtsavdaris@gmail.com

Constantinos Constantinides, MD

ckonstan@med.uoa.gr

Laiko Hospital, Agiou Thoma 17, Athens 11527, Greece 